\title{
CLÚSTER DE TURISMO DE SALUD EN COLOMBIA: REFERENTES PARA LA COMPETITIVIDAD
}

\author{
CLUSTER OF HEALTH TOURISIM IN COLOMBIA: REGARDING \\ COMPETITIVENESS
}

\author{
TÍTULO CORTO: CLÚSTER DE TURISMO DE SALUD EN COLOMBIA
}

\section{Edwin Causado-Rodríguez ${ }^{1}\left(\mathbb{D}\right.$, Amira Mojica-Cueto $^{2}$, Arturo Charris-Fontanilla ${ }^{3}$}

\begin{abstract}
Tipología: Artículo de revisión
Para citar este artículo: Causado-Rodríguez E, Mojica-Cueto A, Charris-Fontanilla A. Clúster de turismo de salud en Colombia: referentes para la competitividad. Duazary. 2018 septiembre; 15 (3): 307-323. Doi: http://dx.doi. org/10.21676/2389783X.2423
\end{abstract}

Recibido en agosto 11 de 2017

Aceptado en septiembre 15 de 2017

Publicado en línea en mayo 30 de 2018

\section{RESUMEN}

El presente artículo presenta parte de los resultados del proyecto de investigación "Estrategias para el incremento de la competitividad mediante la conformación del Clúster de Turismo de Salud en el Área Metropolitana de Bucaramanga -AMB", requisito para optar al título de Magíster en Desarrollo Empresarial de la Facultad de Ciencias Empresariales de la Universidad del Magdalena. El objetivo del presente escrito consiste en evidenciar los avances de la conformación autónoma del clúster de turismo de salud en Colombia, evaluando metodológica y rigurosamente las perspectivas del mercado de turismo en salud a partir de revisión bibliográfica en inglés y español de carácter institucional público, privado y científico por iniciativa empresarial, y de estudios realizados en regiones de Colombia y a nivel mundial, que han implementado este renglón como opción de desarrollo mediante el aprovechamiento de ventajas competitivas circundantes. Entre los resultados obtenidos se muestran con precisión los ángulos o dimensiones de este fenómeno en el contexto económico y social; además, se destacan los patrones de turismo y, a partir de estos, las razones por las cuales se viene avanzando en la conformación de los diferentes clústeres a nivel nacional, permitiendo apreciaciones profundas en el tema a partir de la visión multisectorial de interés.

Palabras clave: mercado de atención de salud; industria del turismo; aglomeración; turismo médico; eficiencia organizacional.

1.Universidad del Magdalena. Santa Marta, Colombia. Correo: ecausado@unimagdalena.edu.co - https://orcid.org/0000-0001-6258-2307

2. Promioriente SAS. Santa Marta, Colombia. Correo: amiraesther@hotmail.com - http://orcid.org/0000-0001-5751-3657

3. Universidad del Magdalena, Santa Marta, Colombia.Correo: arturocharris@gmail.com - https://orcid.org/0000-0003-1160-0485 


\section{ABSTRACT}

This article presents part of the results of the research project "Strategies for Increasing Competitiveness through the Conformation of the Health Tourism Cluster in the Metropolitan Area of Bucaramanga -AMB", required to qualify for the Master's degree in Business Development of the Faculty of Business Sciences of Universidad del Magdalena. The objective of this paper is to demonstrate the progress of the autonomous conformation of the health tourism cluster in Colombia. In this regard, we assess, in a methodological and rigorous way, the prospects of the health tourism market, from bibliographic review in English and Spanish -of a public, private and scientific institutional nature- by business initiative, as well as from studies carried out in regions of Colombia and worldwide, that have implemented this line as a development option by exploiting surrounding competitive advantages. Among the obtained results, the angles or dimensions of this phenomenon, in the economic and social context, become clear. In addition, the tourism patterns are highlighted, as well as the reasons why progress has been made in the conformation of the different clusters at a national level. This allowed deep insights into the issue, based on the interest multisectoral vision.

Keywords: health care market; tourism industry; crowding; medical tourism; organizational efficiency.

\section{INTRODUCCIÓN}

$E^{\prime}$ turismo es considerado uno de los sectores económicos con mayor capacidad para el impulso del desarrollo y crecimiento económico de una región, pues está comprobado que en los lugares turísticos se crea una mayor aglomeración de empresas y de actividades económicas diversas. Esto permite forjar empleos de buena calidad, además de generar efectos multiplicadores de mayor amplitud que otras actividades en los componentes socioeconómicos ${ }^{1,2}$. En este sentido, el turismo de salud se define como el proceso mediante el cual una persona viaja para recibir servicios de salud en un país diferente a aquel en el que reside ${ }^{3-5}$.

En este orden de ideas, una modalidad de turismo es la que se ha denominado el turismo de salud. Este ha sido calificado como sector emergente de clase mundial en el proceso de transformación productiva de Colombia, constituyéndose en un importante renglón de la economía, tanto en el espacio nacional como internacional. Adicionalmente, el turismo de salud surge como una respuesta al deterioro y a las deficiencias de los distintos sistemas de salud en el mundo (grandes tiempos de espera para la atención, envejecimiento de la población, insuficiencia de capacidades, altos costos, baja capacidad asistencial, reducción del nivel de ingresos de la población, población sub asegurada, entre otros $)^{4,5}$.

Entre los objetivos primordiales del presente documento está describir de manera sucinta y precisa el sector de turismo en salud en los mercados internacionales y en Colombia, además de las connotaciones de su funcionamiento en el contexto económico y social, las tendencias de los patrones de turismo de salud y, a partir de estas, las razones que inciden en la conformación autónoma de los diferentes clúster a nivel nacional, como una aglomeración de empresas de los sectores turísticos y de salud asociadas a través de la prestación de servicios de salud.

\section{MATERIALES Y MÉTODOS}

Se realizó una revisión bibliográfica profunda de documentos publicados en inglés y español entre 2007 y 2016, referente a las temáticas de turismo de salud, clúster de turismo de salud, competitividad del clúster de turismo de salud y determinantes de clúster de turismo de salud, 
a nivel nacional e internacional, mediante selección de documentos técnicos institucionales del Departamento Nacional de Planeación, documentos CONPES, el Ministerio de Comercio, Industria y Turismo, cámaras de comercio de Bogotá, Medellín, Cali, Bucaramanga y Barranquilla, documentos de la CEPAL, tesis de grado y artículos científicos de la base de datos ELSEVIER, Science Direct, Web of Science, Scopus, Pubmed, Scielo, Redalyc y Google Académico, con el objeto de obtener la mayor cantidad de información veraz y actualizada sobre la temática seleccionada.

Seguidamente, se revisó cada uno de los documentos hallados, de los cuales se extrajo información estadística y en otros casos información teórica y conceptual, que permitieron evaluar la importancia, la utilidad y la novedad de los estudios, así como la coherencia interna de los mismos y el conocimiento generado, a fin de disminuir los errores que pudieran presentarse durante el procesamiento de los indicadores.

\section{RESULTADOS Y DISCUSIÓN}

\section{Estrategia de clúster para la competitividad}

El concepto de clúster se define como la aglomeración natural de las empresas concentradas en un área geográfica determinada, además de que los actores involucrados comparten complementariedades e interdependencias en el marco de un eslabón productivo en la cadena de valor de interés ${ }^{6-10}$. Seguidamente, se afirma que el nacimiento de un clúster se puede dar a partir de relaciones urbanas y rurales, nacionales e internacionales y de acuerdo a la forma de conexión de las empresas, asegurando a la vez una forma de comunicación explicita y de acuerdo a intereses compartidos ${ }^{11,12}$.

En la perspectiva de los autores de este documento, el clúster se puede definir como la inicia- tiva de consolidación de la oferta de servicios y/o bienes de manera competitiva en una región geográfica, articulando la organización socioproductiva de los distintos actores públicos, privados y de generación de conocimiento a través de vínculos de cooperación y competencia en el largo plazo. Desafortunadamente, la articulación espontánea de los oferentes de bienes y/o servicios de salud y turísticos, como es este caso, no resulta posible; razón por la cual las iniciativas de políticas públicas son determinantes para el logro del propósito de trabajo conjunto y de articulaciones intersectoriales, que de otra manera dificultan la comunicación, la conexidad, la especialización de la economía, la generación de valor, la maximización de beneficios y la disminución de costos.

En este sentido, resulta inobjetable admitir que el turismo de salud a nivel mundial ha venido creciendo con tasas del orden entre 15\% y $30 \%$ en cada una de las categorías que lo conforman (medicina curativa, preventiva, estética y bienestar $)^{13-15}$. Respecto a los destinos con mayor preferencia por turistas internacionales, estos son Latinoamérica y Oriente Medio ${ }^{5,6,8}$. Para la categoría de bienestar (Inspired Wellness), entre los destinos más demandados están Asia y Norteamérica ${ }^{16-18}$. A su vez, en el caso de la oferta Latinoamericana, esta presenta fortaleza en la categoría de medicina estética; Europa para la categoría de rehabilitación; Asia en la categoría de cardiología y, finalmente, Oriente Medio en la categoría de oncología ${ }^{14-16}$.

Para el caso de América Latina se presentan dos aspectos de gran relevancia en la elección de su oferta: precios bajos con respecto a la competencia y la cercanía geográfica a Estados Unidos y Canadá, y mercados con mayor capacidad de pago en la región ${ }^{8,17,19}$. Incluso, se evidencia un incremento de pacientes y acompañantes de $25 \%$ a $30 \%$ y de ingresos entre un $37 \%$ y un $40 \%$ anualmente $\mathrm{e}^{7,16,20}$ (Tabla 1). 
Tabla 1. Comparativo de precios (US\$) en procedimientos médicos por país 2009

\begin{tabular}{|l|c|c|c|c|c|}
\hline \multicolumn{1}{|c|}{ Procedimiento } & Colombia & EE.UU & México & Costa Rica & India \\
\hline Bypass de Corazón & 14.630 & 144.000 & 20.000 & 25.000 & 8500 \\
\hline Angioplastia & 7106 & 57.000 & 16.000 & 13.000 & 8500 \\
\hline $\begin{array}{l}\text { Reemplazo Válvula } \\
\text { del Corazón }\end{array}$ & 10.450 & 170.000 & 30.000 & 30.000 & 12.000 \\
\hline Reemplazo Cadera & 8360 & 50.000 & 13.125 & 12.500 & 8000 \\
\hline Reemplazo de Rodilla & 7106 & 50.000 & 10.650 & 11.500 & 7000 \\
\hline Implantes Dentales & 1672 & 2000 & 910 & 1000 & 700 \\
\hline Banda Gástrica & 6500 & 30.000 & 8430 & 8500 & 7500 \\
\hline Implantes Mamarios & 2600 & 10.000 & 8000 & 3500 & 4500 \\
\hline Rinoplastia & 1677 & 8000 & 4165 & 5500 & 3500 \\
\hline Estiramiento Facial & 3305 & 15.000 & 7200 & 5900 & 7000 \\
\hline
\end{tabular}

Fuente: Cálculos de los autores con base en ${ }^{5,6,8}$.

Por su parte, Colombia representa el rango más bajo en costos de procedimientos médicos, pues la diferencia oscila entre $55 \%$ y $90 \%$, comparándola con los países de la tabla 1 , aunque en horas de viaje es uno de los más altos respecto a sus competidores cercanos. En este sentido, la industria del turismo en salud ya ha sobrepasado los US\$60.000 millones de dólares anuales en ventas y genera el 2,5\% del total mundial de viajes por avión. En total, más de 50 millones de viajeros en el 2011 y un crecimiento sostenido del $20 \%$ al $25 \%$ anual $^{7,16,21}$.

\section{Oferta de servicios de turismo de salud a nivel internacional}

A nivel mundial se viene generando un aumento sostenido de los costos en servicios de salud. En el caso estadounidense, el 18\% de los 253 millones de asegurados en el 2007 no clasifican para ciertos procedimientos médico/quirúrgicos, por lo cual deben buscar financiación propia para los servicios no cubiertos ${ }^{7,16,22}$. Para el caso de los países asiáticos, la India se encuentra catalogado como el país con mayor crecimiento en el turismo de salud. Este país desde 1990 inició un proceso de desregulación de la economía a través de la implementación de algunas medidas de política como la disminución de barreras para la inversión en desarrollo tecnológico e incentivos tributarios, sumado a que el país contaba con una gran cantidad de profesionales médicos educados en Estados Unidos, el Reino Unido y Australia con alto nivel de bilingüismo y una sofisticación tecnológica y de infraestructura. Para el 2009 contaba con 11 hospitales acreditados por la Joint Commission International $\mathrm{JCI}^{16,17}$, por lo que 180 mil turistas de salud llegaron a la India en el 2005 con ingresos por más de US\$350 millones. El incremento de turismo de salud cada año es del 25\%, siendo el país de mayor crecimiento por este concepto ${ }^{16,23}$. 
En segundo orden se presenta Filipinas, el cual también inicio su proceso de promoción del turismo en el área de la salud hacia el 2004, mediante un proyecto liderado por el gobierno, en asocio con el sector privado, denominado "Programa de Turismo Médico de Filipinas -PMPT-. Los resultados para Filipinas comenzaron a notarse desde el año 2006, fecha en la cual entraron 250.000 pacientes no residentes y hubo un ingreso de US\$850 millones de dólares; además, es reconocido por trasplantes y cuidado renal. Las importaciones de equipos médicos al país fueron del orden de los US\$119 millones en el 2005, representando un $32 \%$ más que en $2004^{7,16}$.

En tercer lugar se destaca Tailandia, proyectando tanto al sector público como al privado la exploración del negocio de atención a turistas de salud. Por parte del gobierno se recibieron ayudas de USAID para el Thailand Competitiveness Initiative (2005 - 2006), cuyo objetivo es el mejoramiento de la competitividad y el desarrollo de clúster, entre ellos el de turismo, y dentro de este el de turismo de salud, obteniendo un crecimiento anual de 30\% entre 2001 y 2006. Para el 2005, el gobierno tailandés realizó inversiones por US $\$ 2,98$ millones, brindando instancias y ambientes cómodos para el visitante. Así mismo, logró la certificación por JCI de cinco hospitales, e ingresos por el orden de los US\$1300 millones de dólares ${ }^{7,16}$.

Se concluye que, del liderazgo ejercido por países asiáticos, junto con Norte América son los que atraen el mayor flujo de turistas de salud en busca de servicios en las categorías de medicina, especialmente cardiología, cirugía general y ortopedia (87\%); y un (73\%) para la categoría de cirugía estética por parte de Medio Oriente y Latinoamérica ${ }^{4,9,17}$.

\section{Demanda de servicios de turismo de salud a nivel internacional}

En lo que tiene que ver con la demanda, el análisis arroja que los turistas que buscan turismo en salud con tecnología más avanzada, en un 38\% son de América Latina, 35\% del Medio Oriente, $16 \%$ de Europa, $7 \%$ de Norte América y $4 \%$ de Asia. Respecto a aquellos que buscan mejor calidad, vienen de Asia en un 44\%, Medio Oriente 24\%, Europa 13\%, África 13\%, América Latina $5 \%$ y otros $1 \%$. Los que lo hacen por un acceso más rápido, principalmente en ortopedia y cirugía general, son Reino Unido 27\%, Canadá 21\%, Japón 20\%, Australia 18\% y otros $14 \%$. En cuanto a aquellos turistas que buscan menores costos para procedimientos agudos no críticos con un protocolo estándar a nivel mundial, provienen en un 99\% de Estados Unidos, de los cuales el $61 \%$ se dirigen a Asia, un 36\% a América Latina, 3\% al Medio Oriente y el $1 \%$ a Europa. Aquellos turistas que buscan procedimientos electivos a bajo costo, también provienen de EE.UU. un 30\%, Australia 20\%, Reino Unido 6\%, Hong Kong 4\%, Taiwán 4\%, Japón $3 \%$ y otros $27 \%{ }^{5,7,16,24}$.

\section{América Latina en la perspectiva internacional del turismo de salud}

El turismo médico en esta región de América Latina ha venido tomando fuerza hasta el punto de superar países como Tailandia e India que, como se analizó anteriormente, son catalogados como dos de los países de mayor desarrollo del ramo.

Se destacan países como Costa Rica que atrae a pacientes norteamericanos por su cercanía (alrededor de 2,5 horas en avión desde Miami) $y$ al que se le suman otros elementos como que la segunda lengua es el inglés y que existe una alta afinidad y receptividad del pueblo costarri- 
cense hacia los americanos; la estabilidad política y la buena infraestructura de servicios de turismo se aúna a que además cuenta con dos hospitales acreditados por $\mathrm{JCI}^{7,17,20}$. En el caso de México también se destaca la cercanía a los Estados Unidos, permitiendo a miles de personas encontrar el tratamiento médico cerca de casa, especialmente los de Texas y California. El costo, en comparación con EE.UU., está entre el 25\% y $35 \%$ menos. Cuenta con siete hospitales acreditados por la JCI. Con relación a Sur América, Brasil tiene una gran estructura médico-hospitalaria y equipos de última generación. Brasil cuenta con 13 hospitales y servicios de salud acreditados por la JCI, sus costos están entre el 50\% y el 60\% menos que en EE. UU. y el turismo en este país crece a un ritmo del $10 \%$ anual $^{10,19,21}$.

De acuerdo con la especialidad, a Latinoamérica acuden turistas básicamente por Cirugía Plástica en un $31 \%$, Ortopedia el 15\%, Oncología el 11\%, Cirugía general el 8\% y Cardiología el 6\%, mientras que el continente asiático tiene una menor concentración de especialidades ${ }^{7,16}$.

\section{El clúster de turismo de salud: sector de clase mundial en Colombia}

Desde el año 2007, el Ministerio de Comercio Industria y Turismo de Colombia viene desarrollando el Programa de Transformación Productiva -PTP- enfocado en fortalecer el turismo de salud como un sector de clase mundial; igualmente, dentro del marco de políticas públicas, se establece como política sectorial posicionar a Colombia como un destino de turismo de salud de clase mundial ${ }^{6,14,18,25-27}$.

En efecto, el sistema de salud colombiano se encontraba posicionado en el año 2000 como el de mejor desempeño en el mundo en desarrollo, ubicándose a la altura de Suecia o Bélgica y superando incluso los sistemas de salud de los Estados Unidos y Canadá, ocupando además el puesto 22 a nivel mundial ${ }^{5,25}$. De acuerdo a los rangos de medición del riesgo regulatorio (siendo uno el más bajo y 10 el más alto), Colombia se encuentra con una puntuación de 4,7 ; en cuanto al número de habitaciones de hotel (/1000 personas), la relación en Colombia es del 1,5; el número de camas de hospital (/1.000 personas) es de 1,3; y el índice de accesibilidad (tiempo de desplazamiento hacia el país, frecuencia de vuelos y diferencias horarias respecto a Estados Unidos) es de 4,7 horas $^{5,15,20,26}$.

Colombia hace parte de los cinco principales destinos de bienestar en América Latina junto con México, Argentina, Brasil y Puerto Rico. Dentro de dicho grupo solo posee el 13\% de Spas con respecto a México, que es el primer destino. En el país existen 41 instituciones clasificadas para realizar procedimientos complejos, localizadas especialmente en Bogotá, Medellín, Cali, Bucaramanga y Barranquilla ${ }^{5,15,20}$, con datos de incremento en un $40 \%$ del porcentaje de huéspedes extranjeros no residentes, en hoteles por concepto de turismo en salud y bienestar en el primer cuatrimestre del 2012 comparado con el año $2011^{6,14,18,25-27}$.

En 2008 entraron a Colombia 44.800 pacientes, de los cuales la mayor proporción la constituyen colombianos residentes en EE. UU, dado que un $29 \%$ estuvo motivado por la calidad, $21 \%$ por la tecnología, $21 \%$ por los costos, $21 \%$ por oportunidad y un $8 \%$ por otros; además, el tipo de especialidad más buscada fue la cardiovascular con un $41 \%$, le sigue la bariátrica con $10 \%$, operaciones generales $7 \%$ y oncología con 6\%. En el 2011 arribaron 20.500 pacientes frente a 14.500 que ingresaron en el $2010^{10,14,16}$ (Figuras 1 y 2 ). 
Figura 1. Segmentación de los turistas de Salud en Colombia por tipo de motivación. Fuente: Calculo Autores ${ }^{14-16}$.

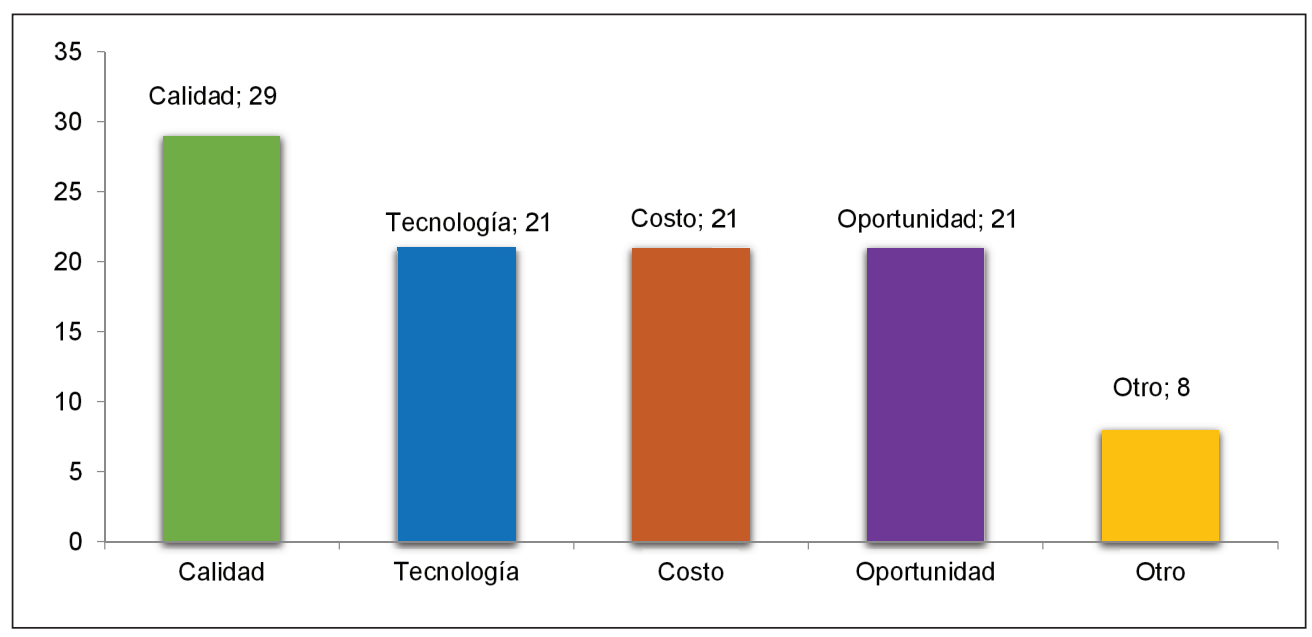

Figura 2. Segmentación de los turistas de Salud en Colombia por tipo de especialidad. Fuente: Calculo Autores $^{14-16}$.

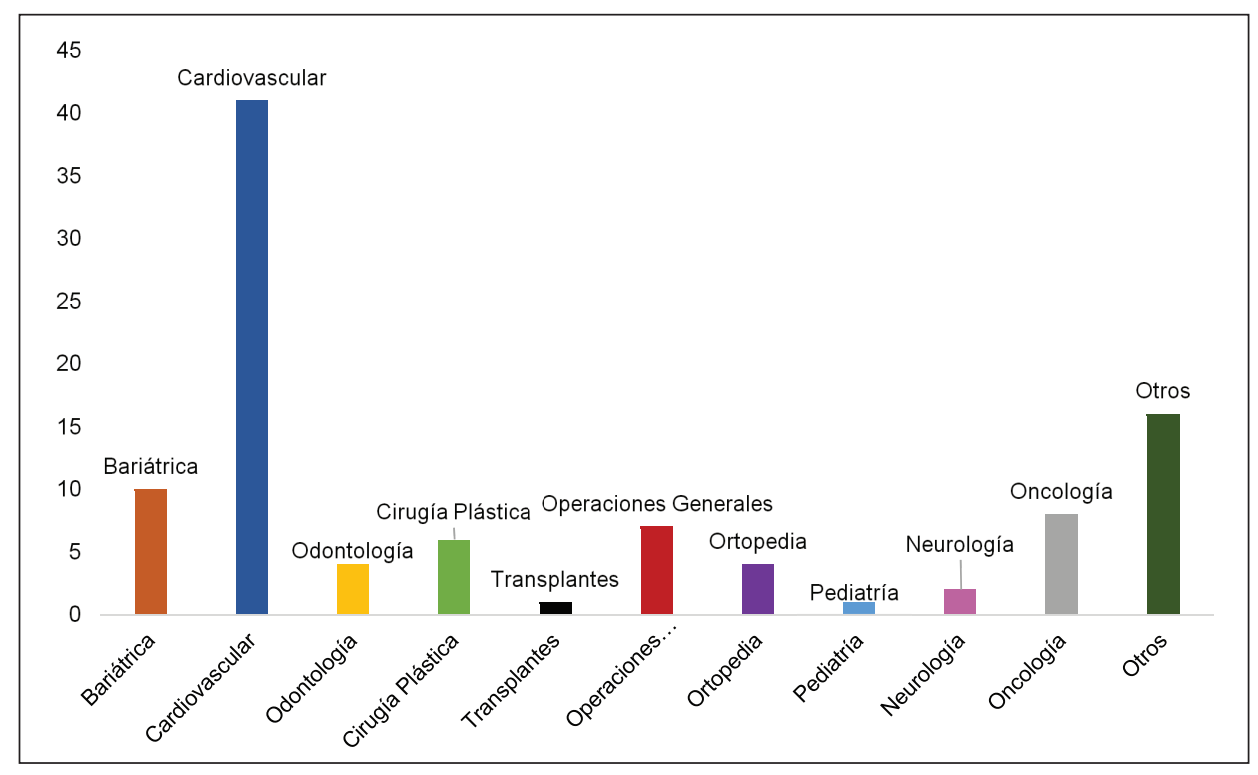

De manera concreta, Colombia cuenta con una buena dotación de infraestructura privada de reputación internacional, con niveles moderados de equipamiento, pero con fuerte concentración en Bogotá, Cali y Medellín. Además, destaca por mayores ventajas en la calificación del recurso humano para poder atender un potencial crecimiento de la demanda internacional y cuenta con tres instituciones con el sello de calidad Joint Commission International, USA -JCI al 2016,5,17. En este sentido, se manifiesta que existen en el mundo alrededor de nueve acreditadores internacionales con mayor prestigio. Entre estos están: 1. JCI- Joint Commission International, 2. NCQANational Committee for Quality Assurance, 3. ESQH- European Society for Quality in Healthcare, 
4. ISQUA- The International Society for Quality in Health Care, 5. Trent Accreditation Scheme (UK) ${ }^{22}$; y se debe elegir, para certificarse, cualquiera de estas, de acuerdo al mercado de interés y al sistema organizacional de la entidad.

\section{Lineamientos estructurales para los clústeres regionales de turismo de salud en Colombia}

Actualmente en Colombia se está generando infraestructura tendiente a la competitividad internacional mediante creación de zonas francas de salud aprobadas por la DIAN, ubicadas en los departamentos de Risaralda, Atlántico, Nariño, Santander, Bolívar, Antioquia y Cundinamarca. Hay otras dos en proceso. En lo que tiene que ver con las políticas y regulaciones, en Colombia se han hecho ingentes esfuerzos para adaptar la normatividad a la promoción de los servicios de salud exportables ${ }^{19,28,29}$. En este sentido, se presenta una primera aproximación del clúster de turismo de salud y la agregación de valor respectiva a lo largo de esta (Figura 3).

Figura 3. Esquematización del Clúster de Turismo de Salud en Colombia.

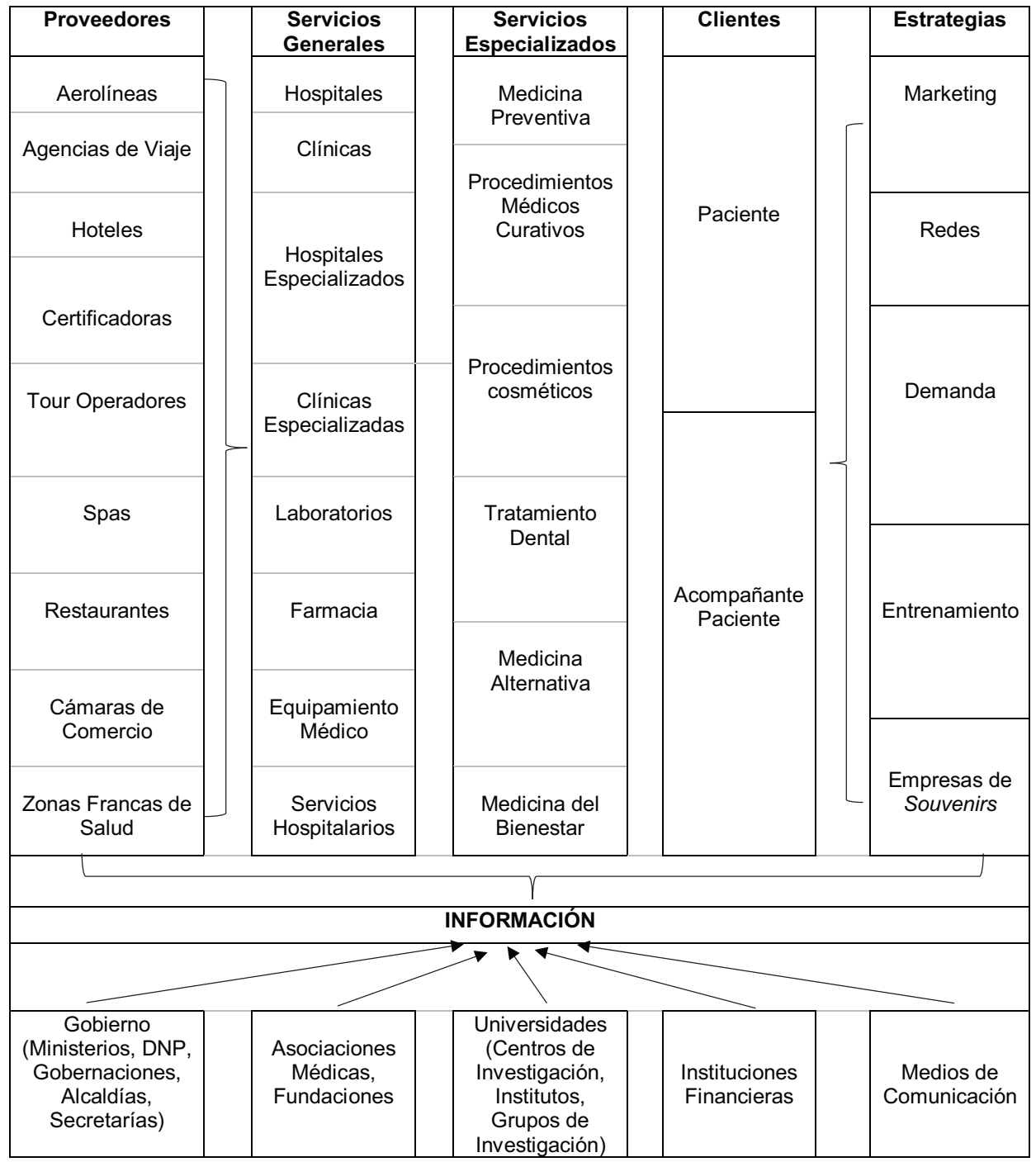

Fuente: Autores. 
Adicionalmente, se plantean estrategias tendientes a generar las condiciones necesarias para garantizar la competitividad del turismo en salud en el país, así: 1. Generación de un marco legal único que responda a las necesidades del entorno. 2. Incremento de los niveles de productividad del sector salud. 3. Fortalecimiento comercial del sector salud mediante la identificación de los oferentes y demandantes. 4. Construcción de un sistema de información liderado por el sector público y privado. 5. Realización del diagnóstico, adecuación física y adquisición de tecnología para la habilitación y la acreditación de las instituciones de salud; y 6. Promoción y consolidación de clústeres de salud regionales para el fortalecimiento interno y favorecer las exportaciones de servicios de salud ${ }^{5,6,14,15,30}$.

\section{Clústeres regionales de turismo de salud en Colombia}

Una de las formas de fortalecer la competitividad del turismo de salud para la exportación es mediante la conformación de clústeres ${ }^{29,31-35}$; para esto se vienen adelantando iniciativas exitosas en el país, entre las que se destaca Medellín por su exitosa experiencia. Desde el año 2009 inauguró el Clúster de Servicios de Medicina y Odontología y la consolidación del proyecto Salud Sin Fronteras, conformado por un grupo de instituciones que, a través de la marca Medellín Healt City, buscan el reconocimiento internacional como uno de los mejores distritos de salud en América Latina ${ }^{28,36-38}$ (Tabla 2).

Tabla 2. Características del Clúster de Turismo de Salud de Medellín

\begin{tabular}{|c|c|c|}
\hline & Entidades del Sector Salud & $\begin{array}{l}\text { Entidades Institucionales } \\
\text { Regionales }\end{array}$ \\
\hline Principales Actores & $\begin{array}{l}\text { Hospital Pablo Tobón Uribe, Clínica Cardiovascular Santa } \\
\text { María, Clínica Medellín, Hospital Universitario San Vicente } \\
\text { de Paúl, Clínica El Rosario, Clínica CES, Clínica Noel, } \\
\text { Clínica Las Vegas, Clínica las Américas, Emergencia Médica } \\
\text { Integral (EMI), Salud Sin Fronteras, Clínica El Rosario, } \\
\text { Hospital General de Medellín, Clínica Clofán, Dental Center, } \\
\text { Clínica Odontológica, Bocca Odontología Contemporánea. }\end{array}$ & $\begin{array}{l}\text { Alcaldía de Medellín y } \\
\text { Cámara de Comercio de } \\
\text { Medellín Para Antioquia, } \\
\text { Gobernación de Antioquia, } \\
\text { Pro-Antioquia. }\end{array}$ \\
\hline Principales actividades & \multicolumn{2}{|c|}{$\begin{array}{l}\text { Promoción de la internacionalización de servicios de salud, acreditación internacional de } \\
\text { las Instituciones Prestadoras de Servicios de Salud -IPS-, fortalecimiento empresarial de la } \\
\text { cadena productiva; y promoción, desarrollo e implementación de un modelo de atención } \\
\text { para la atención de pacientes internacionales. Definición y posicionamiento de la marca } \\
\text { Medellín Health City }{ }^{9} \text {. }\end{array}$} \\
\hline Facturación Promedio & \multicolumn{2}{|c|}{$\begin{array}{l}\text { US } \$ 1.115 \text { millones a 2015. (Prestación de Servicios Clínicos, Odontológicos y Productos y } \\
\text { Servicios Para la Salud Clínica - Farmacia-Prótesis). }\end{array}$} \\
\hline $\begin{array}{l}\text { Hospitales y/o Clínicas } \\
\text { Rankeadas }\end{array}$ & $\begin{array}{l}\text { Reconocimiento y promoción de Medellín como un destino } \\
\text { de salud en Latinoamérica por parte de "Medical Tourism } \\
\text { Association -MTA" (EE. UU.). } \\
\text { - Cinco clínicas en el Ranking Latinoamericano de Mejores } \\
\text { Clínicas y Hospitales } 2016 \text { (Revista América Economía }{ }^{39} \text { ). } \\
\text { - Tres hospitales en el Ranking Latinoamericano de Mejores } \\
\text { Clínicas y Hospitales } 2016 \text { (Revista América Economía). }\end{array}$ & $\begin{array}{l}\text { Acreditación Internacional } \\
\text { American Association for } \\
\text { Accreditation of Ambulatory } \\
\text { Surgery Facilities, Inc- } \\
\text { AAAASFI- de ocho clínicas } \\
\text { odontológicas y cinco } \\
\text { clínicas médicas. }\end{array}$ \\
\hline $\begin{array}{l}\text { Hospital y/o Clínica } \\
\text { Certificada por JCI }\end{array}$ & \multicolumn{2}{|l|}{ Certificación por JCI al Hospital Pablo Tobón Uribe. } \\
\hline
\end{tabular}

Fuente: Cálculos Autores ${ }^{26,37-39}$. 
Se menciona que Bogotá viene adelantando este proceso desde el año 2013, a través del proyecto Salud Capital, mediante el cual se creó la red hospitalaria de la capital y se han desarrollado otras estrategias de interés ${ }^{40-43}$ (Tabla 3).

Otro de los clúster en turismo en salud es el adelantado por Cali bajo el nombre de Valle de la Salud, que inició en el año 2008. Las acciones adelantadas por esta iniciativa se han orientado a buscar contactos y relaciones con centros académicos y de C\&T. Internamente, la ciudad tiene todo para atender la demanda que llegue, ya que cuenta con 163 cirujanos plásticos que realizan cerca de 50 mil procedimientos al año, lo cual representa una tasa de 1,5 por cada 100 mil habitantes, entre otras fortalezas ${ }^{10,14,24,26,47}$ (Tabla 4).

Tabla 3. Características del Clúster de Turismo de Salud de Bogotá

\begin{tabular}{|c|c|c|c|}
\hline & Entidades del Sector Salud & Universidades & $\begin{array}{c}\text { Entidades Institucionales } \\
\text { Regionales }\end{array}$ \\
\hline $\begin{array}{l}\text { Principales } \\
\text { Actores }\end{array}$ & $\begin{array}{l}\text { Hospital La Samaritana, Instituto Federico } \\
\text { Lleras Acosta, Santa María, Hospital de } \\
\text { la Misericordia, Instituto Nacional de } \\
\text { Cancerología, Hospital San Ignacio de Bogotá, } \\
\text { Instituto Materno Infantil, Clínica Country, } \\
\text { MEDERI, Clínica del Occidente, Fundación } \\
\text { Santafé de Bogotá, Fundación Cardio Infantil, } \\
\text { Clínica La Sabana, Clínica Shaio, Clínica de la } \\
\text { Mujer, Clínica Avellaneda, Colsanitas Clínica } \\
\text { Universitaria Colombia, Instituto de Ortopedia } \\
\text { Infantil Roosevelt, Clínica Universidad de La } \\
\text { Sabana. }\end{array}$ & $\begin{array}{l}\text { Universidad Javeriana, } \\
\text { Universidad de La } \\
\text { Sabana, Universidad del } \\
\text { Rosario, Universidad } \\
\text { de Los Andes. }\end{array}$ & $\begin{array}{l}\text { Cámara de Comercio } \\
\text { de Bogotá, Alcaldía de } \\
\text { Bogotá, Gobernación de } \\
\text { Cundinamarca. }\end{array}$ \\
\hline $\begin{array}{l}\text { Principales } \\
\text { Actividades }\end{array}$ & \multicolumn{3}{|c|}{$\begin{array}{l}\text { Proyecto "Ciudad Salud”, zonas francas, entre ellas la Clínica los Nogales como la primera zona franca } \\
\text { autorizada en Bogotá, orientada a materializar la exportación de servicios de salud. Por otra parte se } \\
\text { suman instituciones de trayectoria en la ciudad como la Fundación Cardio-infantil, que desde el } 2009 \\
\text { atiende pacientes extranjeros. }\end{array}$} \\
\hline $\begin{array}{l}\text { Facturación } \\
\text { Promedio }\end{array}$ & \multicolumn{3}{|l|}{ No Disponible. } \\
\hline $\begin{array}{l}\text { Hospitales } \\
\text { y/o Clínicas } \\
\text { Rankeadas }\end{array}$ & \multicolumn{3}{|c|}{$\begin{array}{l}\text { - Cuatro clínicas en el Ranking Latinoamericano de Mejores Clínicas y Hospitales } 2016 \text { (Revista } \\
\text { América Economía). } \\
\text { - Tres hospitales en el Ranking Latinoamericano de Mejores Clínicas y Hospitales } 2016 \text { (Revista América } \\
\text { Economía }^{39} \text { ). }\end{array}$} \\
\hline $\begin{array}{l}\text { Hospital } \\
\text { y/o Clínica } \\
\text { Certificada } \\
\text { por JCI }\end{array}$ & \multicolumn{3}{|l|}{ Certificación por JCI a la Fundación Cardio-infantil. } \\
\hline
\end{tabular}

Fuente: Cálculos Autores ${ }^{39,44-46}$. 
Tabla 4. Características del Clúster de Turismo de Salud del Valle del Cauca

\begin{tabular}{|c|c|c|c|}
\hline & Entidades del Sector Salud & Universidades & $\begin{array}{l}\text { Entidades Institucionales } \\
\text { Regionales }\end{array}$ \\
\hline $\begin{array}{l}\text { Principales } \\
\text { Actores }\end{array}$ & $\begin{array}{l}46 \text { hoteles en la ciudad de Cali. Centro de } \\
\text { Implantología y Rehabilitación Oral CRIO, } \\
\text { Hospital UniversitariodelValle, Centro Quirúrgico } \\
\text { de la Belleza, Clínica Los Andes, Clínica Rey } \\
\text { David, Clínica Comfandi Tequendama, Corpus } \\
\text { \& Rostrum, Dentilaser, Fecundar, Instituto de } \\
\text { Urología Especializada, Odontovalle, Oftalaser, } \\
\text { Centro Internacional de Entrenamiento de } \\
\text { Investigaciones Médicas-CIDEIM, Instituto de } \\
\text { Inmunología - Asoclinic, Clínica Oftalmológica, } \\
\text { Centro Médico Imbanaco, Fundación Clínica Valle } \\
\text { del Lili. }\end{array}$ & $\begin{array}{l}\text { Universidad del Valle, } \\
\text { Universidad Santiago } \\
\text { de Cali, Universidad } \\
\text { San Martín, } \\
\text { Universidad Libre y la } \\
\text { Pontificia Universidad } \\
\text { Javeriana de Cali. }\end{array}$ & $\begin{array}{l}\text { Cámara de Comercio de } \\
\text { Cali, Valle Salud S.A., } \\
\text { Alcaldía Municipal de } \\
\text { Cali, Gobernación del } \\
\text { Valle del Cauca }\end{array}$ \\
\hline $\begin{array}{l}\text { Principales } \\
\text { actividades }\end{array}$ & \multicolumn{3}{|c|}{$\begin{array}{l}\text { Denominación como el "Cluster de la Excelencia Clínica del Valle". Formulación de planes exportadores y } \\
\text { elaboración de la imagen corporativa "Valle Salud" a posicionar en los mercados internacionales }\end{array}$} \\
\hline $\begin{array}{l}\text { Facturación } \\
\text { Promedio }\end{array}$ & \multicolumn{3}{|c|}{$\begin{array}{l}\text { US } \$ 3.563 \text { millones a } 2013 \text { (Incluyendo datos de seis empresas farmacéuticas, prestación de servicios de } \\
\text { salud, e insumos para el sector) }\end{array}$} \\
\hline $\begin{array}{l}\text { Hospitales } \\
\text { y/o Clínicas } \\
\text { Rankeadas }\end{array}$ & \multicolumn{3}{|c|}{$\begin{array}{l}\text { En 2010, Fundación Clínica Valle del Lili (ISQua-ICONTEC) }{ }^{39} \\
\text { En } 2016 \text { Ninguna. }\end{array}$} \\
\hline $\begin{array}{l}\text { Hospital } \\
\text { y/o Clínica } \\
\text { Certificada } \\
\text { por JCI }\end{array}$ & \multicolumn{3}{|l|}{ En 2016 Ninguna. } \\
\hline
\end{tabular}

Fuente: Cálculos Autores ${ }^{14,15,27,39,47}$.

Barranquilla viene desarrollando el clúster de salud del Atlántico desde el año 2011. En esta ciudad se vienen adelantando proyectos tales como el de la Zona Franca de Salud liderado por la Clínica Porto Azul, con una inversión chilena y colombiana cercana a los US\$60 millones de dólares, ubicada en Puerto Colombia48-53 (Tabla 5).

Finalmente, se presenta la iniciativa de Clúster de salud en el Área Metropolitana de Bucaramanga - AMB, desde el año 2011, en donde dos IPS lideran el negocio de la venta de servicios de salud a extranjeros: por un lado, la Fundación Cardiovascular, con la puesta en marcha de la zona franca permanente en salud denominada Hospital Internacional de Colombia; y la otra zona franca permanente en salud, FOSUNAB, liderada por la Fundación Oftalmológica de Santander Carlos Ardila Lule -FOSCAL- y la Universidad Autónoma de Bucaramanga UNAB-. También tendrá un hotel con 40 aparta suites $^{7,22,29,54-59}$ (Tabla 6). 
Tabla 5. Características del Clúster de Turismo de Barranquilla

\begin{tabular}{|c|c|c|c|}
\hline & Entidades del Sector Salud & Universidades & $\begin{array}{l}\text { Entidades } \\
\text { Institucionales } \\
\text { Regionales }\end{array}$ \\
\hline Principales Actores & $\begin{array}{l}\text { Procaps, Clínica Porto Azul, Clínica General } \\
\text { del Norte, E.S.E. CARI, Centro Neurológico } \\
\text { del Norte, Clínica Oftalmológica del Caribe, } \\
\text { Centro Oftalmológico Carriazo, Coomeva } \\
\text { EPS, Geotech, Salud Software House. } \\
\text { Fundación para la Investigación Biomédica, } \\
\text { E-culture International, entre otros. }\end{array}$ & $\begin{array}{l}\text { Universidad Simón } \\
\text { Bolívar, Universidad } \\
\text { de la Costa, Universi- } \\
\text { dad del Atlántico, } \\
\text { Universidad del } \\
\text { Norte. }\end{array}$ & $\begin{array}{l}\text { Cámara de Co } \\
\text { mercio de Barran } \\
\text { quilla, Gobernación } \\
\text { del Atlántico y } \\
\text { Alcaldía Distrital. }\end{array}$ \\
\hline Principales actividades & \multicolumn{3}{|c|}{$\begin{array}{l}\text { Una Zona Franca de Salud - Clínica Porto Azul. Producción y distribución de productos } \\
\text { farmacéuticos. Producción y distribución de equipamiento médico. La prestación de servicios } \\
\text { de salud y actividades de investigación en salud. }\end{array}$} \\
\hline Facturación Promedio & \multicolumn{3}{|c|}{$\begin{array}{l}\text { US } \$ 1.145 \text { millones a } 2011 \text { (Incluyendo el sector farmacéutico, proveedores de servicios de } \\
\text { salud y de equipos) }\end{array}$} \\
\hline $\begin{array}{l}\text { Hospitales y/o Clínicas } \\
\text { Rankeadas }\end{array}$ & \multicolumn{3}{|l|}{ Ninguna } \\
\hline $\begin{array}{l}\text { Hospital y/o clínica } \\
\text { Certificada }\end{array}$ & \multicolumn{3}{|l|}{ Ninguna } \\
\hline
\end{tabular}

Fuente: Cálculos Autores ${ }^{48-53}$.

Tabla 6. Características del Clúster de Turismo de Salud de Santander

\begin{tabular}{|c|c|c|c|}
\hline & Entidades del Sector Salud & Universidades & $\begin{array}{l}\text { Entidades } \\
\text { Institucionales } \\
\text { Regionales }\end{array}$ \\
\hline Principales Actores & $\begin{array}{l}\text { Fundación Cardiovascular de Colombia } \\
\text { - FOSCAL, Instituto del Corazón de } \\
\text { Florida Blanca, Fundación Oftalmológica } \\
\text { de Santander - FOSUNAB y el Hospital } \\
\text { Internacional de Colombia - HIC, Hospital } \\
\text { Universitario de Santander y el Hospital } \\
\text { Psiquiátrico San Camilo. }\end{array}$ & $\begin{array}{lr}\text { Universidad } & \text { Autóno } \\
\text { ma de Bucaramanga, } \\
\text { Universidad r de } \\
\text { Santander - } \\
\text { Universidad } \\
\text { Bosque. }\end{array}$ & $\begin{array}{l}\text { Cámara de Comercio } \\
\text { de Bucaramanga, } \\
\text { Gobernación de } \\
\text { Santander, SENA. }\end{array}$ \\
\hline $\begin{array}{l}\text { Principales } \\
\text { actividades }\end{array}$ & \multicolumn{3}{|c|}{$\begin{array}{l}\text { Dos zonas francas especiales en salud: Fundación Oftalmológica de Santander -FOSUNAB y el } \\
\text { Hospital Internacional de Colombia -HIC. Construcción de plataformas tecnológicas y aplicación } \\
\text { de la Telemedicina. }\end{array}$} \\
\hline $\begin{array}{l}\text { Facturación } \\
\text { Promedio }\end{array}$ & \multicolumn{3}{|c|}{ US $\$ 8.000 .000$.oo en 2012, solo datos de una institución Certificada por JCI } \\
\hline $\begin{array}{l}\text { Hospitales y/o } \\
\text { Clínicas Rankeadas }\end{array}$ & \multicolumn{3}{|c|}{$\begin{array}{l}\text { Dos hospitales (Universitario y Privado) en el Ranking Latinoamericano de Mejores Clínicas y } \\
\text { Hospitales } 2016 \text { (Revista América Economía }{ }^{39} \text { ). }\end{array}$} \\
\hline $\begin{array}{l}\text { Hospital y/o clínica } \\
\text { Certificada }\end{array}$ & \multicolumn{3}{|c|}{ Certificación por JCI a la Fundación Cardiovascular de Colombia - FOSCAL. } \\
\hline
\end{tabular}

Fuente: Cálculos Autores ${ }^{7,29,39,54,55}$. 
La tendencia en la prestación de servicios de turismo de salud se encuentra en dos grandes vertientes, tales como la medicina curativa y de bienestar, en donde se pretende curar una enfermedad de forma rápida y segura y ofrecer bienestar al acompañante del paciente respectivamente. En este sentido, la creación de valor de manera aproximada en el área de medicina curativa se distribuye en un $15 \%$ en honorarios médicos, $45 \%$ hospitalización, 30\% medicinas e insumos y $10 \%$ estudios médicos. Para el caso de la medicina estética, el $70 \%$ es de honorarios médicos, $10 \%$ hospitalización, $15 \%$ medicina e insumos y $5 \%$ comisión intermediarios ${ }^{7,10,14-16}$. Estas cifras indican que la cantidad de intervenciones en medicina estética son mayores que la medicina curativa y de bienestar; sin embargo, estas dos últimas generan mayores ingresos al inversionista y al sector en general.

Finalmente, de todas las categorías del turismo de salud, la medicina de bienestar es la que genera los mayores ingresos, por lo cual se recomienda mayor incremento en inversiones de spas, tratamientos alternativos y finca raíz asociada al turismo de gran auge en la actualidad.

\section{CONCLUSIÓN}

Se puede concluir que la estrategia de clústeres regionales de turismo de salud en Colombia genera competitividad en ambos sectores, dado que presenta una gran fortaleza en inversiones de primer orden, mayor dinamismo económico de manera sostenida, además de aspectos de gran interés como la interrelación de esfuerzos entre el sector público, el sector privado y la academia, para lograr una fuerte inversión tecnológica y de infraestructura, servicios de alta calidad a bajo costo, talento humano disponible y altamente capacitado, y una figura jurídica y tributaria (zonas francas de salud) que garantizan la inversión y la retribución de esta.
Sin embargo, en los aspectos técnicos y financieros se evidencia a lo largo del documento que, de todas las categorías de turismo de salud, la medicina de bienestar es la que genera los mayores retornos y que presenta tendencia creciente, por lo cual se recomienda mayor incremento en inversiones de spas, tratamientos alternativos y finca raíz asociada al turismo de gran auge en la actualidad, debido a que se viene apostando fuertemente en el sector por la cirugía estética, la cual tiene altas demandas, pero bajos niveles de rentabilidad.

Finalmente, si bien es cierto que esta iniciativa del clúster de turismo de salud se encuentra implementada en los departamentos mencionados, realmente no se encuentra consolidada. Es evidente que falta mayor articulación entre el sector hotelero, las aerolíneas, las agencias de viajes, las asociaciones médicas y la academia, mediante desarrollo de iniciativas conjuntas que aporten a la consolidación de esta, tales como paquetes de servicios conjuntos, aplicaciones tecnológicas de agregación de información y monitoreo, y diseño de estrategias de desarrollo integrales que apunten al aprovechamiento de las oportunidades presentes en el entorno y se adelanten a las posibles amenazas que surgen constantemente en el desarrollo empresarial.

\section{RECOMENDACIONES}

Finalmente, de manera explícita se recomienda avanzar en aspectos comola definición de normas formales e informales de estructuras administrativas del clúster, así como en la consolidación de proyectos conjuntos regionales e intersectoriales entre el sector turismo y el sector salud, gestión de ruedas de negocios para inversionistas nacionales e internacionales y disponibilidad de recursos en $\mathrm{I}+\mathrm{D}+\mathrm{I}$ con el fin de mejorar la eficiencia productiva del sector, establecer sistemas de información que permitan monitorear 
avances, dificultades y mejoras del sector, definir propuesta de valor diferenciado para el acompañante del demandante del sector de turismo de salud; consolidar protocolos, manuales y procedimientos estandarizados que garanticen la calidad en la prestación del servicio, avanzar en los procesos de certificación internacional de JCI a fin de acceder a pacientes del mercado de Estados Unidos y Canadá con mayor capacidad de pago y establecer modelo de negocio institucional por parte del estado a mediano y largo plazo, a fin de construir de manera compartida una misión y visión sectorial.

\section{DECLARACIÓN SOBRE CONFLICTOS DE INTERESES}

Todos los autores participaron en el diseño, análisis e interpretación de datos. No presentan ningún conflicto de intereses relacionado con esta contribución al campo de conformación de clúster de turismo de salud. De igual forma, todos los autores son independientes con respecto a las instituciones financiadoras y de apoyo, y durante la ejecución del trabajo o la redacción del manuscrito no incidieron intereses o valores distintos a los que usualmente tiene la investigación.

\section{REFERENCIAS BIBLIOGRÁFICAS}

1. Arias F, Caraballo A, Matos R. El turismo de salud : Conceptualización, historia, desarrollo y estado actual del mercado global. Clio America. 2012;6(11): 72-98.

2. Arechavala R. Proceso de clusterización en Jalisco. Retos de aprendizaje y la colaboración empresarial. México: Editorial Universitaria; 2014.

3. Barriga A, Farías M, Ruiz A, Sánchez A, Jiménez W. Turismo en salud: una tendencia mundial que se abre paso en Colombia. Ciencia y Tecnología. 2011; 9(1): 125-37.

4. Comisión Económica Para América Látina y el Caribe. Escalafón de Competitividad de los Departamentos de Colombia. 2012-2013. Serie Estudios y Perspectivas - Bogotá No. 27. Santiago de Chile: CEPAL - Naciones Unidas; 2014.

5. Gonzalez-Mendoza J, Fonseca-vigoya M. Cadena de Valor Turismo de Salud del Área Metropolitana de Cúcuta. Respuestas. 2016; 21(1): 28-44.

6. Departamento Nacional de Planeación. Documento CONPES 3678, Política de Transformación Productiva: Un modelo de desarrollo sectorial para Colombia. Bogota: DNP; 2010.

7. Ministerio de Comercio, Industria y Turismo. Convenio de competitividad turística departamento de Santander. Matriz de compromisos. Visión: Destino de aventura, salud y cultura. Bogotá: Mincomercio; 2011.

8. Departamento Nacional de Planeación. Agenta Interna para la Productividad y Competitividad. Documento Regional Santander. Bogota: DNP; 2007.

9. Causado-Rodriguez E, García J, Martínez J, Herrera, A. Tecnologías de Información y Comunicación en el Sector Hotelero. Primera edición. Barranquilla: Editorial Corporación Universitaria Latinoamericana CUL; 2015

10. Farías M.Estudio de Mercado del Turismo de Salud. Análisis e Investigación. Carana Corporation. Empresas y Empleos. 2011; Septiembre.

11. Porter M. La ventaja competitiva de las naciones. Buenos Aires Agentina: Javier Vergara Editores; 1991.

12. Solvell O. Clusters balancing evolutionary and constructive forces. 1a ed. Stockholm, Sweden: Ivory Tower Publishing; 2008. 
13. Porter M. Las cinco fuerzas competitivas que le dan forma a la estrategia. Harvard Business Review - América Latina; 2008.

14. Ministerio de Comercio, Industria y Turismo. Programa de Transformación Productiva. Sectores de clase mundial. Asi avanza la transofrmación productiva. Consultado $17 \mathrm{de}$ agosto de 2017. En https://www.ptp.com.co/ documentos/informe\%20completo\%20ptp.pdf.

15. Proexport Colombia. Exportando Servicios de Salud. Gerencia de Servicios. 2011. Consultado el 18 de agostode2017. Disponibleen:https://www.minsalud. gov.co/sites/rid/1/Exportaci\%C3\%B3n\%20de\%20 Servicios\%20de\%20Salud.pdf.

16. Ministerio de Comercio, Industria y Turismo. Desarrollando sectores de clase mundial en Colombia. Informe Final Sector Turismo de Salud. Bogotá-Colombia: Mincomercio; 2009.

17. Beladi H, Chao C, Ee M, Hollas D. Medical tourism and health worker migration in developing countries. Economic Modelling. 2015; 46: 391-6.

18. Cámara de Comercio de Medellín para Antioquia. Documentos Comunidad Clúster No. 1: Clúster una estrategia para crear ventaja competitiva. Medellín: Camara de Comercio de Medeín; 2006.

19. Mojica A. Estrategias para el Incremento de la Competitividad Mediante la Conformación del Clúster de Turismo de Salud en el Área Metropolitana de Bucaramanga-AMB. [Trabajo de grado Magister en Desarrollo Empresarial]. Santa Marta: Universidad del Magdalena. Facultad de Ciencias Empresariales y Económicas; 2015.

20. Hernández V, Forero O. Por qué Colombia no es un país líder en la categoría bienestar del sector turismo de salud. [Trabajo de grado Profesional en Negocios Internacionales]. Bogotá: Universidad EAN. Facultad de Estudios en Ambientes Virtuales; 2013.
21. Castro A, Villarraga M, Barreto L, Victoria J, Barbosa G. Turismo en salud: una tendencia mundial que se abre paso en Colombia. Ciencia \& Tecnología para la Salud Visual y Ocular. 2011; 9(1): 125-137.

22. Banco de La Republica. Bucaramanga: capital humano y crecimiento económico. Aguilera M. Documento de Trabajo sobre Economía Regional No. 180. Cartagena: Banco de la República, CEER; 2013.

23. Brubakk K, Vist G, Bukholm G, Barach P, Tjomsland O. A systematic review of hospital accreditation: The challenges of measuring complex intervention effects. BMC Health Services Research. 2015; 15(1): 1-10.

24. Falcone S. Medellín - The Spirit of Latin America. Medical Tourism. 2010; febrero. 14 (enero febrero), 27-31.

25. Cámara de Comercio de Medellín para Antioquia. El turismo de salud en el contexto internacional y las condiciones para el acceso al mercado. Medellín: Cámara de Comercio de Medellín - Politécnico Colombiano Jaime Isaza Cadavid; 2015.

26. Castaño M. Turismo en Salud: Resultados de la sistematización y análisis de las encuestas de satisfacción aplicadas a pacientes extranjeros atendidos en una clínica de Medellín. Intersección. 2014; 1(1): 24-35.

27. Forestieri K. Turismo Médico como opción de desarrollo económico del sector salud en Colombia. [Trabajo de grado Especialización en Administración Hospitalaria]. Bogotá: Universidad EAN. Facultad de Ciencias de La Salud; 2012.

28. Marulanda J, Correa G, Mejía L. El clúster de salud en Medellín, ventaja competitiva alternativa para la ciudad. EAN 67; 2009.

29. McKercher B. Towards a taxonomy of tourism products. Tourism Management. 2016; (54): 196-208. 
30. Shen H, Song C, Li M, Jiang Q. Shaping destination images through social networking sites: A case study of Singapore. Advances in Hospitality and Leisure. 2015; 11: 89-107.

31. Michopoulou E, Buhalis D. Information provision for challenging markets: The case of the accessibility requiring market in the context of tourism, Information \& Management. 2013; 50: 229-239.

32. Woodhead A. Scoping medical tourism and international hospital accreditation growth. International Journal of Health Care Quality Assurance. 2013; 26(8): 688-702.

33. Kachniewska M.Towards the Definition of a Tourism Cluster, Journal of entrepreneurship, management and innovation JEMI. 2013; 9(1): 33-56.

34. Small J, Darcy S, Packer T. The embodied tourist experiences of people with vision impairment: Management implications beyond the visual gaze. Tourism Management. 2012; 33(4): 941-50.

35. Causado-Rodriguez E, Reatiga I. Cadenalogística de subproductos residuales en la industria de tajada de plátano para exportación. Dimensión empresarial. 2013; 11(2): 9-16

36. Cámara de Comercio de Medellín para Antioquia. Documentos Comunidad Clúster No. 2. Inexmoda. Un caso exitoso de liderazgo. Medellín: Cámara de Comercio de Medellín; 2007.

37. Cámara de Comercio de Medellín para Antioquia. Documentos Comunidad Clúster No. 8: Diseño e implementación de un modelo Clúster para la atención de pacientes internacionales en la ciudad de Medellín. Medellín: Camara de Comercio de Medellín; 2013.

38. 38. Gangulia S, Ebrahim A. A qualitative analysis of Singapore's medical tourism competitiveness.
Tourism Management Perspectives. 2017; 21: 74-84.

39. 39. Rankin 2016 Clínicas y Hospitales. Revista América Economía. Revisada 20 de Agosto de 2017. Disponible en: https://rankings. americaeconomia.com/2016/clinicas/ranking

40. Alcaldia Mayor de Bogotá. Indicadores de competitividad del sector turístico de Bogotá: Una propuesta en la perspectiva de la Competitividad Sistémica. Bogota: Instituto Distrital de Turismo - IDT; 2014.

41. Alcaldia Mayor de Bogotá. Estudios previos convenio IDT - Universidad Distrital. Bogota: Instituto Distrital de Turismo - IDT; 2014.

42. Alcaldia Mayor de Bogotá. Estimación de la contribución al PIB y al crecimiento económico de Bogotá. Bogotá: Instituto Distrital de Turismo - IDT; 2014.

43. Alcaldia Mayor de Bogotá. Informe de gestión y resultados 2014. Bogotá: Instituto Distrital de Turismo - IDT; 2014.

44. Vélez J. Determinantes de la inversión en innovación en el sector servicios de Bogotá. Estimaciones Econométricas a Nivel de la Firma. Ensayos Sobre Política Económica. 2009; 27(60): 110-167.

45. Alcaldía Mayor de Bogotá. Clústeres Turísticos en Bogotá: Experiencia y Estudio de Casos. Bogotá: Universidad Distrital Francisco José De Caldas - Alcaldía Mayor de Bogotá - Instituto Distrital de Turismo; 2015.

46. Valencia M. Turismo en Medicina Estética: Ventajas y Desventajas Para Bogotá, Comparada con la Oferta de Medellín. [Trabajo de grado Profesional en Administración de Empresas]. Bogotá: Universidad Militar Nueva Granada. Facultad de Estudios a Distancia - Programa de Administración de Empresas; 2016. 
47. Restrepo L. Clúster de servicios de salud del Valle del Cauca: desarrollo de una oferta competitiva hacia la internacionalización. Cali: Sello Editorial Javeriano; 2008.

48. Padilla E. Factores Determinantes de la innovación en el clúster de salud del Atlántico. [Trabajo de grado Profesional de Administración de Empresas]. Barranquilla: Universidad del Norte. Escuela de Negocios; 2012.

49. Ramos J, Moreno J, Polo J, Almanza C. Clúster en el Caribe colombiano: el caso del Atlántico: desempeño innovador y dinamización de la economía. Barranquilla: Editorial Universidad del Norte; 2014.

50. Hernández R. Determinantes de la innovación en el clúster de obra blanca en el departamento del Atlántico. [Trabajo de grado Profesional en Administración de Empresas]. Barranquilla: Universidad del Norte. Escuela de Negocios; 2012.

51. Torres S. Clúster en salud como estrategia de desarrollo empresarial en Barranquilla (Colombia, Suramérica). Óptica gremial año 2012. Dictamen Libre. 2012; 10/11: 97-110.

52. Mendoza J. La dinámica de los clústeres. Revista Dimensión Empresarial. 2014 Enero - Junio; 12(1): 84-97.

53. Benavides H, Cujía J, Marenco M. El Clúster de Salud del Departamento del Atlántico. [Trabajo de grado Profesional en Administración de Empresas]. Barranquilla: Corporación Universidad de la Costa. Facultad de Ciencias Económicas; 2012.

54. Barajas S, Graz A, Suarez E. Estudio del clúster de la salud en Santander EPS, IPS a partir de la Ley 100. Compendios de Administración. Análisis Competitivo. 2009 Enero; (1): 25-41.

55. Lunta N, Horsfalla D, Hanefeld J. Medical tourism: A snapshot of evidence on treatment abroad. Maturitas. 2016 Jun; (88): 37-44.

56. 56. De la Puente M. Sector del turismo de salud: Caso de Colombia. Economía del Caribe. 2015; (16): 129-61.

57. Viloria EJ, Bertel NM, Daza CA. Percepciones estudiantiles sobre el proceso de acreditación por alta calidad del Programa de Administración de Empresas de la Universidad del Magdalena. Praxis. 2015; 11: 89-102.

58. Ureña VY, Jiménez PI, Mejía AA, Vilarete MJ. Cultura innovativa: prospectiva de las condiciones estratégicas para el desarrollo del capital humano. Clío América. 2014; 8(16): 195-200

59. Sanclemente TJ. La responsabilidad social empresarial (RSE) de los pequeños distribuidores minoristas. Clío América. 2015; 9(17): 42-56. 